\title{
Inductance Plethysmography: An Alternative Signal to Servocontrol the Airway Pressure during Proportional Assist Ventilation in Small Animals
}

\author{
ANDREAS SCHULZE, CLEIDE SUGUIHARA, TILO GERHARDT, PETER SCHALLER, \\ NELSON CLAURE, RUTH EVERETT, CARLOS DEVIA, DOROTHY HEHRE, AND \\ EDUARDO BANCALARI \\ Department of Pediatrics, Division of Neonatology, University of Miami, Miami, Florida, U.S.A. \\ [A.S., C.S., T.G., P.S., N.C., R.E., C.D., D.H., E.B.]; and the Department of Obstetrics and Gynecology, \\ Division of Neonatology, Ludwig Maximilian University of Munich, Munich, Germany [A.S.]
}

\begin{abstract}
During proportional assist ventilation (PAV), the ventilator pressure is servocontrolled throughout each spontaneous inspiration such that it instantaneously increases in proportion to the airflow (resistive unloading mode), or inspired volume (elastic unloading mode), or both (combined unloading mode). The PAV pressure changes are generated in a closed-loop feedback circuitry commonly using a pneumotachographic signal. In neonates, however, a pneumotachograph increases dead space ventilation, and its signal may include a substantial endotracheal tube leak component. We hypothesized that respiratory inductive plethysmography (RIP) can replace pneumotachography to drive the ventilator during PAV without untoward effects on ventilation or respiratory gas exchange. Ten piglets and five rabbits were supported for 10-min (normal lungs) or 20-min (meconium injured lungs) periods by each of the three PAV modes. In each mode, three test periods were applied in random order with the ventilator driven by the pneumotachograph signal, or the RIP abdominal band signal, or the RIP sum signal of rib cage and abdomen. Interchanging the three input signals did not affect the
\end{abstract}

\section{ABSTRACT}

regularity of spontaneous breathing, and gas exchange was achieved with similar peak and mean airway pressures (ANOVA). However, the RIP sum signal worked adequately only when the relative gains of rib cage and abdominal band signal were calibrated. We conclude that an RIP abdominal band signal can be used to generate PAV, avoiding increased dead space and endotracheal tube leak problems. (Pediatr Res 49: 169-174, 2001)
Abbreviations:
AB, RIP signal from abdominal band
CPAP, continuous positive airway pressure
$\mathrm{Paco}_{2}$, arterial $\mathrm{PcO}_{2}$
$\mathrm{Pao}_{2}$, arterial $\mathrm{Po}_{2}$
PAV, proportional assist ventilation
RC, RIP signal from rib cage band
RIP, respiratory inductive plethysmography
Sum, sum signal of AB and RC
TCD, total compartmental displacement

During PAV, the applied positive airway pressure is servocontrolled throughout each spontaneous inspiration such that it increases in proportion to a weighted sum of the airflow and inspired volume of spontaneous breathing (1-4). During expiration, the airway pressure returns to a preset positive endexpiratory pressure level. Thus, the inspiratory change in airway pressure has two components: a resistive unloading component that increases the airway pressure in proportion to inspiratory airflow (5), and an elastic unloading component

Received March 7, 2000; accepted August 18, 2000

Correspondence and reprint requests: Andreas Schulze, M.D., Ludwig Maximilian University, Klinikum Grosshadern, Department of Obstetrics and Gynecology, Division of Neonatology, Marchioninistrasse 15, D-81377 Munich, Germany.

Supported in part by the University of Miami Project New Born. that additionally increases the airway pressure in proportion to the inspiratory volume (6). These two components decrease resistive and elastic work of breathing in proportion to their gain settings (7).

To accomplish the proportional changes in airway pressure, the ventilator uses airflow and volume signals from a pneumotachograph as reference to generate the pressure waveforms. The pneumotachograph is mounted between the ventilator circuit and the endotracheal tube. This sensor provides clear, artifact-free signals in most cases. However, in neonates and particularly in small premature infants, the use of a pneumotachograph may be associated with a significant additional dead space load. Moreover, its signal may include a substantial and varying endotracheal tube leak component. This mimics 
inspiratory airflow and renders the signal useless for generating pressure waveforms in proportion to changes in lung volume. A small flow sensor is prone to malfunction whenever condensed water, secretions, or other particles obstruct its flow channels. Furthermore, the airflow signal as measured at the proximal airway may lag behind the diaphragmatic effort in the presence of substantial chest wall distortion. In this situation, the rise in ventilator pressure during PAV may be unduly delayed.

This study evaluates the applicability of RIP signals as substitutes for pneumotachograph signals to provide input information for volume- or flow-PAV. Inductance plethysmography derives respiratory volume changes noninvasively from inductance coils wrapped around the chest and abdomen (810). This system does not add any respiratory dead space, and the signal is not influenced by endotracheal tube leaks. However, it may be prone to body motion artifacts (11).

We hypothesized that RIP signals can replace pneumotachography as input signal to the ventilator during PAV. As markers for assessing the effectiveness of PAV with the different input signals, we measured arterial blood gases, tidal volume, minute ventilation, airway and esophageal pressure changes, and indices of thoracoabdominal motion asynchrony. Piglets and adult rabbits with normal and meconium-injured lungs were studied to test the system across different configurations and sizes of chest and abdomen, across different breathing patterns, and with normal and impaired pulmonary mechanics.

\section{METHODS}

Animal model. Ten newborn piglets $<10$ d old (weight, $3120 \pm 603 \mathrm{~g}$ [mean $\pm \mathrm{SD}])$ and five young adult New Zealand White rabbits $(3140 \pm 891 \mathrm{~g})$ were anesthetized with $2 \mathrm{mg} / \mathrm{kg}$ xylazine and $20 \mathrm{mg} / \mathrm{kg}$ ketamine intramuscularly. Anesthesia was maintained by a continuous i.v. sodium pentothal infusion of $3-5 \mathrm{mg} / \mathrm{kg} / \mathrm{h}$. This dosage suppressed gross body movements but not spontaneous breathing efforts. Intravenous infusion fluids were administered at $3 \mathrm{~mL} / \mathrm{kg} / \mathrm{h}$. Tracheostomy was performed on each animal, and the trachea was cannulated with an endotracheal tube (length, $150 \mathrm{~mm}$; inner diameter $3.0 \mathrm{~mm}$ for piglets, $2.5 \mathrm{~mm}$ for rabbits), secured in place by a peritracheal ligature. An arterial catheter was inserted into the femoral artery (piglets) or the right carotid artery (rabbits) for intermittent determination of arterial blood gases (Ciba-Corning Diagnostics Corp., Medfield, MA, U.S.A.). A fluid-filled $8 \mathrm{~F}$ infant feeding tube was inserted orally for esophageal manometry. The tip of the catheter was first advanced into the stomach and then withdrawn while observing the on-line pressure tracing until the largest negative inspiratory pressure deflections occurred. The catheter was flushed intermittently to maintain patency and to prevent gas bubbles from accumulating. The pressure transducer (Sorenson Transpac Transducers, Abbott Critical Care Systems, North Chicago, IL, U.S.A.; precision higher than $\pm 0.01 \mathrm{kPa}$, amplitudefrequency response flat to $20 \mathrm{~Hz}$ ) was calibrated with a water manometer as reference. The animals were then placed in a right decubitus position and connected to a ventilator. They received $100 \% \mathrm{O}_{2}$ throughout the study.

The transducer bands for RIP (Respigraph Noninvasive Monitoring Systems, Miami Beach, FL, U.S.A.) were cloth of $2.5 \mathrm{~cm}$ width with embedded sinusoid coils of Teflon-insulated wire. The rib cage band was wrapped around the chest wall with its lower rim $1 \mathrm{~cm}$ above the manubrium sterni. The upper rim of the abdominal band was placed $2 \mathrm{~cm}$ below the costal margin. The position of the bands was secured with tape. We started the Respigraph and used its built-in qualitative diagnostic calibration procedure to set the relative electrical gains of the rib cage and abdominal transducers $(9,12)$. The protocol was then commenced for the study on normal animals.

To induce lung injury, two doses of $2 \mathrm{~mL} / \mathrm{kg}$ freshly prepared $20 \%$ human meconium in sterile saline solution were introduced deep into the trachea while the animals were rotated from side to side to assure even distribution. Between doses, the animals were manually ventilated with a bag. Meconium solution that did not distribute into the periphery of the lung was removed by repeated endotracheal suctioning. Conventional time-cycled, pressure-limited mechanical ventilation was then initiated for stabilization. Starting $30 \mathrm{~min}$ after meconium application during conventional ventilation, blood gases were obtained at 10-min intervals. The peak inflation pressure was adjusted if the $\mathrm{Paco}_{2}$ was outside a target range of 30-50 $\mathrm{mm} \mathrm{Hg}$. Additional doses of $0.5-1 \mathrm{~mL} / \mathrm{kg}$ meconium solution were given until the $\mathrm{PaO}_{2}$ values no longer improved between blood sampling intervals. A maximum of up to $3 \mathrm{~mL} / \mathrm{kg}$ of additional meconium solution was required in some animals. The degree of lung injury was judged stable enough for the purpose of the experiment when changes in $\mathrm{Pao}_{2}$ and $\mathrm{Paco}_{2}$ levels were $<15 \%$ and did not show any consistent trend for at least $30 \mathrm{~min}$. The protocol was then repeated to study the animals with injured lungs. The Respigraph was recalibrated immediately before restarting the protocol.

Protocol. The animals were exposed to 10-min (normal lungs) or 20-min (injured lungs) epochs of PAV with three types of settings: 1) elastic unloading, 2) resistive unloading, and 3) combined unloading. Each type was applied three times with different input signals to the ventilator: 1) the pneumotachograph signal, 2) the AB, and 3) the Sum. The gain of the flow-pressure and the volume-pressure feedback, i.e. the degree of unloading, was adjusted to achieve equal peak inspiratory pressures within each mode. The end-expiratory pressure was set to 0 before lung injury and increased to $0.2 \mathrm{kPa}$ thereafter. At the end of each test period, airflow, airway pressure, esophageal pressure, and the inductance signals were recorded over at least 10 consecutive respiratory cycles. Arterial blood samples were then obtained. Before the next test setting, a sidehole of $1 \mathrm{~mm}$ in diameter was opened in the endotracheal tube connector for a period of five respiratory cycles to mimic an endotracheal tube leak (Fig. 1). This was done to document the different consequences of a leak on ventilator pressure waveform when using the pneumotachograph or the inductance plethysmography signals as input. To allow for sequence effects, all test settings were applied in random order determined by choosing from a set of nine sealed envelopes that contained the mode assignment and the input 

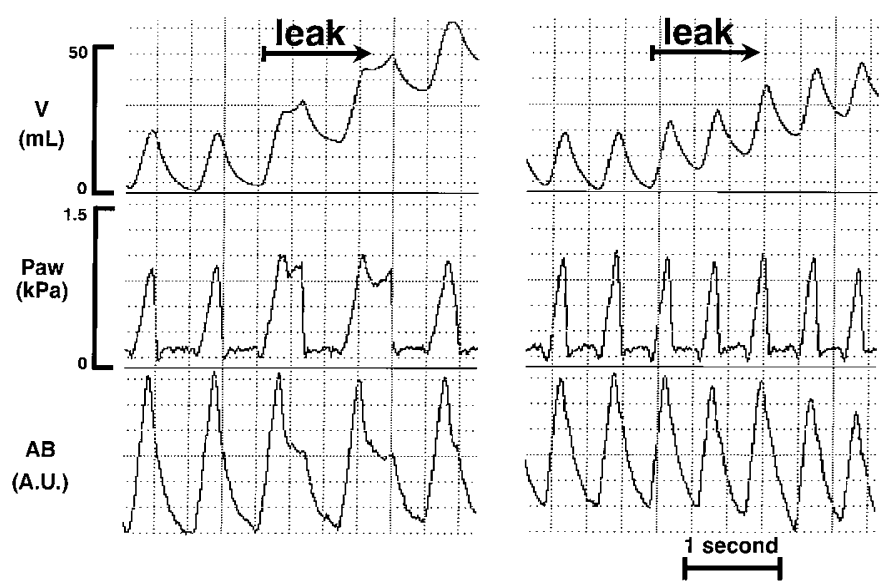

Fig. 1. Volume-PAV (elastic unloading) in a piglet with meconium-injured lungs. The airway pressure (Paw) waveforms were generated on the basis of a pneumotachographic signal (left) or from an AB (right). An endotracheal tube leak was opened at the beginning of the third breath. The airway pressure waveforms remain unaffected by the leak when based on the inductance signal (right). They are distorted and out of proportion to the volume entering the lung when based on the pneumotachographic signal (left). Upper ventilator pressure and inspiratory time limits were in place to prevent excessive and prolonged rise in airway pressure during leaks. $V$, volume of spontaneous breathing as measured by a pneumotachograph mounted between the endotracheal tube and the $\mathrm{Y}$ connector of the respirator circuit; A.U., arbitrary units.

signal type to the ventilator. To preclude potential carryover effects, at least 5 min of CPAP (normal lungs) or conventional mechanical ventilation (postmeconium lungs) were interposed between the test runs.

This protocol was approved by the University of Miami Institutional Review Board.

Ventilator. A STEPHANIE Infant Ventilator (F. Stephan Medizintechnik GmbH, Gackenbach and Dresden, Germany) was used throughout the study. Technical details of this device have been described elsewhere (13). It is a servocontrolled system with sensors for airway pressure and airflow near the endotracheal tube. The flow sensor is a pneumotachometer with a $1.1 \mathrm{kPa} / \mathrm{L} / \mathrm{s}$ resistance at $5 \mathrm{~L} / \mathrm{min}$ flow and a dead space of $0.9 \mathrm{~mL}$. In addition to the conventional modes of mechanical ventilation, the STEPHANIE Infant Ventilator provided elastic unloading, resistive unloading, or a combination of these modalities. During the latter modes, the ventilator receives the flow signal of spontaneous breathing as input to a closed-loop rapid feedback circuitry that generates a pressure at the endotracheal tube connector as output. This pressure is proportional to the inspiratory flow signal (resistive unloading), or the integral of the flow signal, i.e. the volume signal (elastic unloading), or the sum of the two (combined resistive and elastic unloading). The proportions between the outlet pressure and the flow or volume signals can be adjusted as gains on continuous scales in $\mathrm{kPa} / \mathrm{L} / \mathrm{s}$ or $\mathrm{kPa} / \mathrm{mL}$, respectively. For the purpose of this study, the ventilator was modified so that the user could substitute the flow signal of the pneumotachograph for other analog electrical signals, such as the plethysmograph signals, as input to the feedback circuitry. The pneumotachograph remained in place for recording purposes when the other driving signals were used.
Data acquisition and processing. The analog signals of airflow, airway pressure, esophageal pressure, and inductance plethysmography were digitized at a rate of $100 \mathrm{~Hz}$ and recorded on disk using data acquisition software (DATAQ Instruments, Inc., Akron, OH, U.S.A.). All the recorded signals were displayed on-line on the computer screen during the experiments. Tidal volume was obtained by digital integration of flow. The TCD ratio was calculated as the ratio between the sum of the absolute values of rib cage and abdominal displacement (entering a negative chest wall movement as positive) divided by the true sum of those values (8). The TCD increases above 1.0 with chest wall distortion. A delay (lag) between the start of inspiratory airflow and the beginning of the outward movement of the chest wall from its resting position was measured in degrees of the total respiratory cycle.

Statistics. Data are given as mean \pm SD. Repeated measures ANOVA was used to compare data obtained with the three input signals under investigation (pneumotachograph signal, $\mathrm{AB}$, and Sum from inductance plethysmography). Bonferroni's method was applied to isolate differences among the three signal types. Significance was accepted for $p<0.05$. To provide a measure of the reproducibility of the measurements, coefficients of variation were calculated for the within animal data variability from CPAP periods interposed between the PAV episodes.

\section{RESULTS}

Pulmonary compliance and resistance as measured before starting the protocol were $22 \pm 9 \mathrm{~mL} / \mathrm{kPa} / \mathrm{kg}$ and $6.8 \pm 2.4$ $\mathrm{kPa} / \mathrm{L} / \mathrm{s}$, respectively, in piglets; $23 \pm 7 \mathrm{~mL} / \mathrm{kPa} / \mathrm{kg}$ and $5.2 \pm$ $0.6 \mathrm{kPa} / \mathrm{L} / \mathrm{s}$, respectively, in rabbits. Five piglets died during meconium application. Postmeconium pulmonary compliance and resistance were $8 \pm 3 \mathrm{~mL} / \mathrm{kPa} / \mathrm{kg}$ and $7.9 \pm 4.0 \mathrm{kPa} / \mathrm{L} / \mathrm{s}$, respectively, in the remaining piglets; $10 \pm 3 \mathrm{~mL} / \mathrm{kPa} / \mathrm{kg}$ and $7.0 \pm 0.8 \mathrm{kPa} / \mathrm{L} / \mathrm{s}$, respectively, in rabbits. The reproducibility of the physiologic measures as assessed during baseline CPAP periods is given in Table 1.

The resistive unloading part of the protocol could not be completed in any of the animals after meconium injury because arterial oxygenation could not be maintained with the low end-inspiratory airway pressures that occurred with resistive unloading alone. The animals maintained a regular breathing

Table 1. Coefficients of variation for the within animals data variability, calculated from $C P A P$ periods between the $P A V$ episodes

\begin{tabular}{lccccc}
\hline \multirow{2}{*}{\multicolumn{1}{c}{ Variable }} & \multicolumn{2}{c}{ Piglets } & & \multicolumn{2}{c}{ Rabbits } \\
\cline { 2 - 3 } \cline { 5 - 6 } & $\begin{array}{c}\text { Normal } \\
(n=10)\end{array}$ & $\begin{array}{c}\text { Postmeconium } \\
(n=5)\end{array}$ & & $\begin{array}{c}\text { Normal } \\
(n=5)\end{array}$ & $\begin{array}{c}\text { Postmeconium } \\
(n=5)\end{array}$ \\
\hline Tidal volume & $5.4 \pm 2.1$ & $4.3 \pm 3.5$ & & $3.7 \pm 1.4$ & $2.2 \pm 0.8$ \\
Respiratory rate & $3.7 \pm 1.0$ & $3.9 \pm 2.5$ & & $7.6 \pm 3.9$ & $1.5 \pm 0.4$ \\
Minute ventilation & $5.1 \pm 1.5$ & $4.6 \pm 2.2$ & & $10.2 \pm 5.1$ & $2.2 \pm 0.9$ \\
Pes & $13.5 \pm 3.6$ & $14.1 \pm 9.9$ & & $18.1 \pm 12.4$ & $16.1 \pm 7.9$ \\
TCD & $3.7 \pm 4.5$ & $7.4 \pm 6.5$ & & $6.3 \pm 5.3$ & $12.0 \pm 8.4$ \\
Lag RC vs AB & $28.4 \pm 19.1$ & $20.1 \pm 11.7$ & $41.0 \pm 18.8$ & $22.6 \pm 15.7$ \\
\hline
\end{tabular}

Mean \pm SD across the studied animals. Coefficients of variation for individual animals were calculated as SD divided by the mean in percent. Pes, amplitude of tidal esophageal pressure deflection. 
pattern during all other test settings before and after meconium injury. Their arterial blood gases were not significantly different among the three input signals. With few exceptions, the outcome variables reflecting ventilation and the pressures generated by the ventilator were also similar with the different input signals (data on elastic unloading are given in Tables 2 and 3, data on resistive and combined unloading are available from the authors on request). The few statistically significant differences between settings are small in size and may be regarded as clinically insignificant. None of these small differences were consistent in both species or in both the normal and injured lung situations. Chest wall distortion as measured by the TCD ratio and by the lag time was small in all normal animals. It increased moderately after meconium aspiration. Significant differences in these indices between input signals did not occur during any of the PAV test runs (Tables 2 and 3). There was never a consistent time lag of measurable size between the onset of inspiratory airflow as measured by the pneumotachograph and the onset of the inspiratory outward movement of the abdomen.

When a leak around the endotracheal tube developed, airway pressure waveforms generated from a pneumotachograph signal became distorted and out of proportion to the true volume and airflow changes of spontaneous breathing. In contrast, pressure waveforms generated from plethysmography signals were not affected by leaks around the endotracheal tube (Fig. 1).
Visual inspection of the ventilator pressure waveforms did not show qualitative differences between input signals. Plots of tidal volume versus ventilator pressure generated with plethysmography signals as feedback showed a linear relationship (Fig. 2). However, ventilator pressure waveforms driven by the plethysmographic Sum became asynchronous to the animals' breathing pattern when two conditions coincided: chest wall distortion and inadequate calibration of the plethysmograph. In the presence of chest wall distortion and an inappropriately high gain of the $\mathrm{RC}$ relative to the $\mathrm{AB}$, the plethysmographic Sum may show a decrease or remain flat during initial inspiration instead of showing a rise. The decline of Sum during expiration may then be delayed because the rib cage signal becomes positive during expiration. This, in turn, delays the rise in airway pressure at the beginning of inspiration and retards its decline around the transition from inspiration to expiration (Fig. 3). Such signs of inappropriate relative calibration of the rib cage and abdominal sensors occurred regularly after dislocation of the bands during gross body movements or after major changes in body position. They were not noted initially after the plethysmography device had passed the standard calibration procedure.

\section{DISCUSSION}

A PAV device generates immediate airway pressure changes proportional to an input signal of flow, volume, or both. These

Table 2. Comparison of gas exchange, ventilatory variables, and indices of chest wall distortion in normal animals during volume-PAV generated from a pneumotachograph signal or from RIP

\begin{tabular}{|c|c|c|c|c|c|c|}
\hline \multirow[b]{2}{*}{ Variable } & \multicolumn{3}{|c|}{ Piglets $(n=10)$} & \multicolumn{3}{|c|}{ Rabbits $(n=5)$} \\
\hline & PT & $\mathrm{AB}$ & Sum & PT & $\mathrm{AB}$ & Sum \\
\hline $\mathrm{PaO}_{2}(\mathrm{~mm} \mathrm{Hg})$ & $451 \pm 52$ & $456 \pm 39$ & $446 \pm 51$ & $492 \pm 29$ & $463 \pm 22$ & $453 \pm 42$ \\
\hline $\mathrm{PaCO}_{2}(\mathrm{~mm} \mathrm{Hg})$ & $31.8 \pm 4.1$ & $30.2 \pm 3.8$ & $31.3 \pm 4.9$ & $47.1 \pm 15.7$ & $47.2 \pm 14.2$ & $49.3 \pm 13.3$ \\
\hline Tidal volume $(\mathrm{mL} / \mathrm{kg})$ & $7.3 \pm 1.4$ & $7.2 \pm 1.5$ & $7.5 \pm 1.6$ & $5.2 \pm 1.6$ & $5.1 \pm 1.5$ & $4.6 \pm 0.8$ \\
\hline Respiratory rate (breaths/min) & $66 \pm 21$ & $67 \pm 18$ & $59 \pm 22 *$ & $34 \pm 6$ & $36 \pm 7$ & $37 \pm 1$ \\
\hline Minute ventilation $(\mathrm{mL} / \mathrm{kg} / \mathrm{min})$ & $462 \pm 92$ & $473 \pm 137$ & $418 \pm 116$ & $183 \pm 91$ & $186 \pm 81$ & $173 \pm 32$ \\
\hline Mean airway pressure $(\mathrm{kPa})$ & $0.16 \pm 0.07$ & $0.15 \pm 0.05$ & $0.16 \pm 0.07$ & $0.10 \pm 0.05$ & $0.09 \pm 0.05$ & $0.10 \pm 0.04$ \\
\hline Peak inspiratory pressure $(\mathrm{kPa})$ & $0.65 \pm 0.13$ & $0.71 \pm 0.16$ & $0.65 \pm 0.13$ & $0.51 \pm 0.16$ & $0.50 \pm 0.14$ & $0.49 \pm 0.14$ \\
\hline Pes $(\mathrm{kPa})$ & $1.25 \pm 0.65$ & $1.18 \pm 0.54$ & $1.25 \pm 0.61$ & $0.48 \pm 0.15$ & $0.47 \pm 0.13$ & $0.52 \pm 0.15$ \\
\hline TCD & $1.12 \pm 0.17$ & $1.09 \pm 0.1$ & $1.14 \pm 0.19$ & $1.04 \pm 0.02$ & $1.08 \pm 0.05$ & $1.08 \pm 0.04$ \\
\hline Lag $\mathrm{RC}$ vs $\mathrm{AB}$ (degrees) & $48 \pm 42$ & $58 \pm 41$ & $56 \pm 51$ & $48 \pm 16$ & $59 \pm 31$ & $64 \pm 35$ \\
\hline
\end{tabular}

PT, pneumotachography; Pes, amplitude of tidal esophageal pressure deflection.

* Significantly different from PT (repeated measures ANOVA, Bonferroni’s $t$ test). Values are mean \pm SD.

Table 3. Comparison of gas exchange, ventilatory variables, and indices of chest wall distortion in meconium-injured animals during volume-PAV generated from a pneumotachograph signal or from RIP

\begin{tabular}{|c|c|c|c|c|c|c|}
\hline \multirow[b]{2}{*}{ Variable } & \multicolumn{3}{|c|}{ Piglets $(n=5)$} & \multicolumn{3}{|c|}{ Rabbits $(n=5)$} \\
\hline & PT & $\mathrm{AB}$ & Sum & PT & $\mathrm{AB}$ & Sum \\
\hline $\mathrm{PaO}_{2}(\mathrm{~mm} \mathrm{Hg})$ & $271 \pm 138$ & $261 \pm 151$ & $246 \pm 131$ & $175 \pm 117$ & $168 \pm 136$ & $227 \pm 93$ \\
\hline Tidal volume $(\mathrm{mL} / \mathrm{kg})$ & $7.9 \pm 2.2$ & $6.9 \pm 1.8$ & $6.5 \pm 1.9 *$ & $4.3 \pm 0.3$ & $4.4 \pm 0.4$ & $4.0 \pm 0.2$ \\
\hline Respiratory rate (breaths/min) & $70 \pm 26$ & $78 \pm 27$ & $69 \pm 25$ & $113 \pm 31$ & $119 \pm 31$ & $112 \pm 46$ \\
\hline Minute ventilation $(\mathrm{mL} / \mathrm{kg} / \mathrm{min})$ & $508 \pm 160$ & $509 \pm 133$ & $413 \pm 115$ & $480 \pm 132$ & $523 \pm 138$ & $435 \pm 150 \dagger$ \\
\hline Pes $(\mathrm{kPa})$ & $1.34 \pm 0.45$ & $1.35 \pm 0.50$ & $1.37 \pm 0.41$ & $0.69 \pm 0.22$ & $0.66 \pm 0.25$ & $0.73 \pm 0.30$ \\
\hline $\mathrm{TCD}$ & $1.26 \pm 0.33$ & $1.58 \pm 0.72$ & $1.23 \pm 0.22$ & $2.37 \pm 1.60$ & $2.10 \pm 1.26$ & $1.75 \pm 0.70$ \\
\hline Lag RC vs AB (degrees) & $87 \pm 33$ & $90 \pm 43$ & $78 \pm 35$ & $155 \pm 48$ & $138 \pm 35$ & $175 \pm 59$ \\
\hline
\end{tabular}

* Significantly different from PT; $\dagger$ significant difference between sum and AB; $\$$ significantly different from PT and from AB. Values are mean \pm SD. 


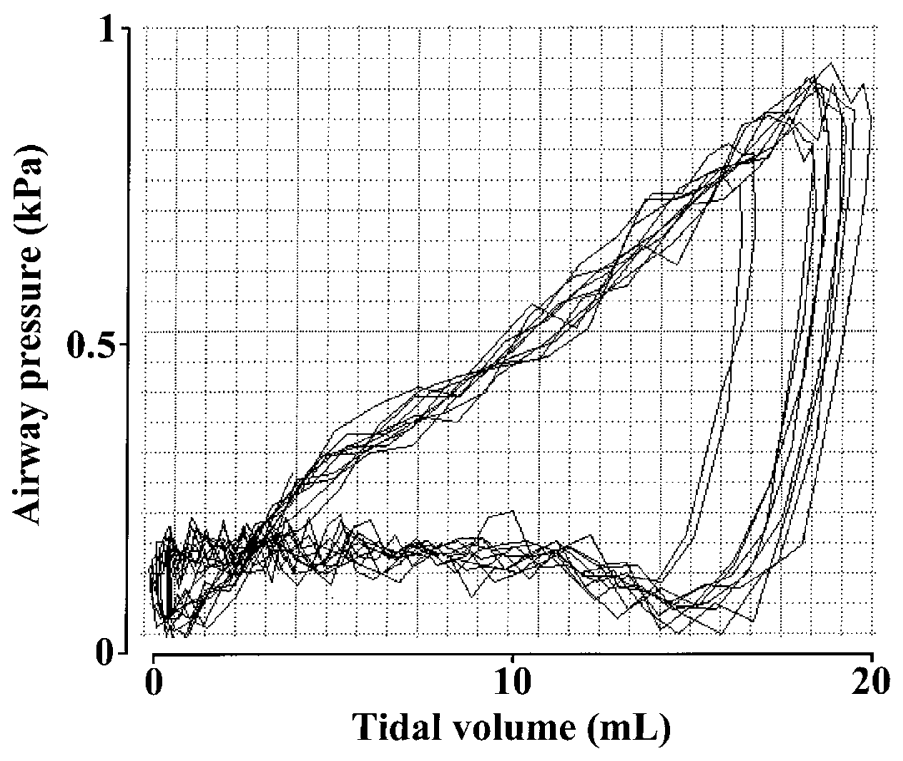

Fig. 2. Ten consecutive breaths during volume-PAV (elastic unloading). The ventilator generated the inspiratory increase in airway pressure with the $\mathrm{AB}$. Tidal volume was measured by pneumotachography at the endotracheal tube. Note that airway pressure is in proportion to tidal volume during inspiration. Loops turn clockwise. Tracing for a piglet with meconium-injured lungs breathing at 96 breaths/min.

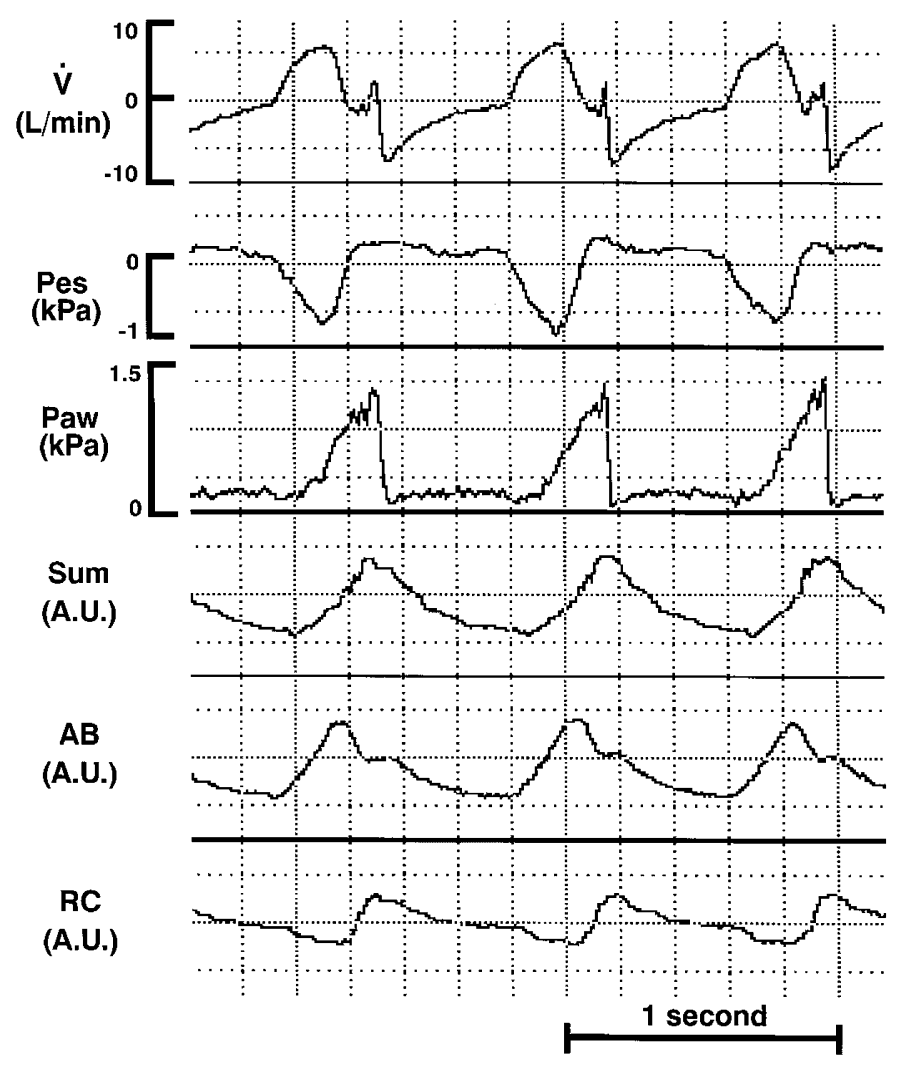

Fig. 3. Volume-PAV mode based on an inappropriately calibrated Sum in a piglet with meconium-injured lungs. Sum is out of phase with the spontaneous respiratory effort because the $\mathrm{RC}$ component has been assigned an inappropriately high gain. As there is inward movement of the rib cage during initial inspiration, the rise of Sum is delayed. This, in turn, causes the airway pressure to lag behind the inspiratory effort. Because the RC becomes positive during expiration, airway pressure continues to rise after inspiration is completed, thus delaying expiration. Pes, esophageal pressure; $V$, airflow. signals are usually obtained by pneumotachography. This study shows that inductance plethysmography can provide a reliable input signal. Arterial blood gases, ventilation, and ventilator pressure changes were similar when the ventilator was driven by the pneumotachography signal compared with the use of plethysmography.

To produce PAV, plethysmography is superior to pneumotachography in at least two respects: it avoids an additional dead space and an overestimation of tidal airflow and volume in the presence of an endotracheal tube leak. Both these aspects are of particular concern in low birth weight infants. Their tidal volumes are small relative to any added dead space such as that of a flow sensor in the airway. They also usually have an endotracheal tube leak, because tightly fitting endotracheal tubes are avoided to prevent laryngeal and tracheal injury. We selected two small animal species for their physiologic characteristics to render the study relevant for infants undergoing neonatal intensive care. The body weight range of our animals covers the weight range of newborn infants. The animals' rapid respiratory rates and small tidal volumes represent typical breathing patterns of term and preterm infants.

PAV driven by a single AB was of sufficient quality to allow for regular breathing, effective ventilation, and gas exchange. This may have important implications for patients without an endotracheal tube on noninvasive ventilation. The usually large and changing leak during noninvasive ventilation would not impair the performance characteristics of a PAV system based on inductance plethysmography. With a single abdominal band, the setup for PAV for those patients would also be simplified compared with the use of other input signals. The standard relative calibration of the two inductance signals was shown to be easily lost during body movements. This calibration, however is redundant when only the abdominal sensor is used. We initially expected a further advantage of the AB in that it might detect the inspiratory effort earlier than a pneumotachograph in the presence of chest wall distortion. This, however, could not be corroborated in our study, possibly because the chest wall in our animals was relatively stable and the induced degree of chest wall distortion was not high enough.

Our study shows that the Sum may not be useful under clinical conditions. An appropriate calibration of the relative weights of the $\mathrm{RC}$ and $\mathrm{AB}$ components of the plethysmographic Sum would be essential in the presence of any degree of chest wall distortion. Otherwise, asynchrony between the airway pressure waveforms and the spontaneous respiratory efforts will reduce the effectiveness of the system. With Sumdriven PAV, there was a trend toward lower tidal volumes and decreased minute ventilation in meconium-injured animals of both species (Table 3). This may be related to inadequate calibration of the plethysmograph during the study. A good calibration, however, is sometimes difficult to obtain, and frequent recalibration may become necessary with a variety of conditions, such as change in sleep state, body position, and dislocation of an inductance plethysmography band (11). Our study was performed on anesthetized animals that were mostly motionless, with quiet or dyspneic, single-posture breathing. This provided an optimal standardized situation for the induc- 
tance system. In clinical practice, additional limitations to the use of plethysmography may arise as bands may change location with frequent gross body movements in active newborn babies. This may compromise the effectiveness of plethysmography for this application and requires further study. It should, however, be less of a safety concern because the PAV software makes provision for gross artifacts on the input signal by limiting peak airway pressures and inflation times, and by providing backup conventional ventilation when no reasonable signal of spontaneous breathing is received.

In conclusion: RIP signals, in particular those from the abdomen alone, can be used to drive a respirator in the proportional assist modes. This offers advantages to infant ventilation as it avoids problems associated with the use of pneumotachographs, such as obstruction of the sensor by secretions or condensed water, additional dead space, and effects of varying endotracheal tube leaks.

\section{REFERENCES}

1. Younes M, Puddy A, Roberts D, Light RB, Quesada A, Taylor K 1992 Proportional assist ventilation: results of an initial clinical trial. Am Rev Respir Dis 145:121-129

2. Younes M 1992 Proportional assist ventilation, a new approach to ventilatory support: theory. Am Rev Respir Dis 145:114-120
3. Poon CS, Ward SA 1986 A device to provide respiratory-mechanical unloading. IEEE Trans Biomed Eng 33:361-365

4. Schulze A, Schaller P, Töpfer A, Kirpalani H 1993 Resistive and elastic unloading to assist spontaneous breathing does not change functional residual capacity. Pediatr Pulmonol 16:170-176

5. Schulze A, Schaller P, Gehrhardt B, Mädler HJ, Gmyrek D 1990 An infant ventilator technique for resistive unloading during spontaneous breathing: results in a rabbit model of airway obstruction. Pediatr Res 28:79-82

6. Schulze A, Schaller P, Jonzon A, Sedin G 1993 Assisted mechanical ventilation using elastic unloading: a study in cats with normal and injured lungs. Pediatr Res 34:600-605

7. Schulze A, Suguihara C, Gerhardt T, Schaller P, Claure N, Everett R, Bancalari E 1998 Effects of respiratory mechanical unloading on thoracoabdominal motion in meconium-injured piglets and rabbits. Pediatr Res 43:191-197

8. Sackner MA, Gonzalez H, Rodriguez M, Belsito A, Sackner DR, Grenvik S 1984 Assessment of asynchronous and paradoxic motion between rib cage and abdomen in normal subjects and in patients with chronic obstructive pulmonary disease. Am Rev Respir Dis 130:588-593

9. Adams JA, Zabaleta IA, Stroh D, Johnson P, Sackner MA 1993 Tidal volume measurements in newborns using respiratory inductive plethysmography. Am Rev Respir Dis 148:585-588

10. Allen JL, Greenspan JS, Deoras KS, Keklikian E, Wolfson MR, Shaffer TH 1991 Interaction between chest wall motion and lung mechanics in normal infants and infants with bronchopulmonary dysplasia. Pediatr Pulmonol 11:37-43

11. Wilkes DL, Revow M, Bryan MH, England SJ 1987 Evaluation of respiratory inductive plethysmography in infants weighing less than 1,500 grams. Am Rev Respir Dis 136:416-419

12. Sackner MA, Watson H, Belsito AS, Feinerman D, Suarez M, Gonzalez G 1989 Calibration of respiratory inductive plethysmograph during natural breathing. J Appl Physiol 66:410-420

13. Schaller P, Schulze A 1991 A ventilator generating a positive or negative internal compliance. Ups J Med Sci 96:219-234 